\title{
Clinical outcome of posterior fixation surgery in patients with vertebral metastasis of lung cancer
}

\author{
TOMOYUKI IGARASHI $^{1 *}$, KEIGO OKAMOTO ${ }^{1 *}$, KOJI TERAMOTO $^{2}$, RYOSUKE KAKU $^{1}$, KEIKO ISHIDA $^{1}$, \\ KEIKO UEDA $^{1}$, YO KAWAGUCHI ${ }^{1}$, TETSUO HORI ${ }^{1}$, MASAYUKI HASHIMOTO ${ }^{1}$, \\ SHOJI KITAMURA ${ }^{1}$, NORIAKI TEZUKA ${ }^{1}$ and JUN HANAOKA ${ }^{1}$
}

Departments of ${ }^{1}$ Surgery and ${ }^{2}$ Medical Oncology, Shiga University of Medical Science, Otsu, Shiga 520-2192, Japan

Received October 3, 2016; Accepted February 17, 2017

DOI: $10.3892 /$ mco.2017.1199

\begin{abstract}
Vertebral metastasis of non-small-cell lung cancer (NSCLC) often leads to neurological paralysis, with deterioration of the patients' activities of daily living (ADL). Surgical treatments for the symptoms are unlikely to be recommended due to the poor prognosis of patients with advanced NSCLC. The aim of the present study was to retrospectively evaluate the clinical outcome of posterior spinal fixation surgery in patients with neurological paralysis resulting from vertebral metastasis of NSCLC. Between April, 2007 and March, 2012, 4 patients (3 men and 1 woman; median age, 56.5 years) underwent fixation surgery at the Shiga University of Medical Science Hospital (Otsu, Japan). The mean preoperative Tokuhashi and Tomita scores of the patients were high (8.25 and 7.0, respectively). However, the Frankel grade functional score and performance status of the patients improved following fixation surgery, after which all patients received chemoradiotherapy. Postoperatively, the median paralysis-free time was 41 months (range, 17-42 months) and the median survival time was 42.5 months (range, 22-43 months). According to the functional scores, the patients had a poor prognosis, which may have been a contraindication for fixation surgery. In these cases, however, surgical treatment improved the patients' ADL and increased the likelihood of receiving anticancer therapy, contributing to the prolongation of survival. Therefore, fixation surgery may be beneficial for patients with neurological paralysis following vertebral metastasis of advanced NSCLC.
\end{abstract}

Correspondence to: Dr Koji Teramoto, Department of Medical Oncology, Shiga University of Medical Science, Seta-Tsukinowa, Otsu, Shiga 520-2192, Japan

E-mail: teramoto@belle.shiga-med.ac.jp

*Contributed equally

Key words: posterior fixation surgery, vertebral metastasis, non-small-cell lung cancer, Tokuhashi score, Tomita score, prognosis, skeletal-related events

\section{Introduction}

Cancer is one of the leading causes of mortality worldwide. In 2012, it was reported that 14 million individuals suffered from cancer and that 8.2 million patients succumbed to the disease. The number of patients who succumbed to lung cancer increased from 1.2 to 1.6 million worldwide between 2000 and 2012 (1). Over the last decade, advances in combined therapeutic modalities have a granted survival benefit to patients with lung cancer. However, the survival of patients who undergo radical surgery for non-small-cell lung cancer (NSCLC) is unsatisfactory, with a median relapse-free survival (RFS) of 52.8 months and a 5-year RFS rate of $47.3 \%$ (2). The main reason for these disappointing figures is that lung cancer easily develops metastases in distant organs, such as the adrenal grand, liver, brain and bone. It is reported that $\sim 60 \%$ of patients with NSCLC already have micro-metastases at initial diagnosis (3), indicating that early detection and treatment of distant metastases is crucial for improving the prognosis of lung cancer patients.

The bones are one of the most common metastatic sites in patients with advanced lung cancer. Previous studies have reported detection of spinal metastases in $\sim$ one-third of autopsied cancer patients (4), and $60 \%$ of patients with lung cancer have been found to have spinal metastases (5). Lung cancer (as well as breast and prostate cancer) is a type of cancer that frequently develops bone metastases, which cause skeletal-related events (SREs) including pain, paralysis, pathological fractures and hypercalcemia (6). These SREs compromise the patients' quality of life (QoL) and activities of daily living (ADL), and may deprive them of the opportunity to receive further anticancer therapy.

Medical treatment of bone metastases includes a bisphosphonate, zoledronic acid, which delays the onset of SREs. In addition, denosumab, a human monoclonal antibody against the receptor activator of nuclear factor $x \mathrm{~B}$ ligand, was reported to significantly delay the time to onset of SREs when compared with zoledronic acid (20.6 vs. 16.3 months, respectively) (7). Surgical treatment for vertebral metastases often reduces symptoms such as pain and progressive paralysis, contributing to improvement in the patients' ADL. These surgical effects may be immediately observed in patients with vertebral metastases. However, surgical 
treatment is only recommended if the patients' expected survival is $>3$ months ( 8 ).

The Tokuhashi score (9) and the Tomita score (10) are used to predict the prognosis of patients with bone metastases; based on these scores, the indications for surgical treatment of bone metastases may be assessed. The criteria of the Tokuhashi score include general condition [performance status (PS)], number of extraspinal bone metastases, number of metastases in the vertebral body, metastases in major internal organs, site of primary cancer and the extent of paralysis according to the Frankel grade functional score (11). The criteria of the Tomita score comprise the aggressiveness of the primary tumor, the presence of visceral metastases and the number of bone metastases.

In Tokuhashi and Tomita scoring, patients with bone metastases from advanced NSCLC are allotted a high score due to the aggressive nature of the NSCLC. Therefore, such patients are unlikely to be considered as candidates for surgical treatment. However, given that these scores do not take into consideration the effect of novel anticancer therapy consisting of moleculartargeted drugs, it was questioned whether these scores are still suitable for determining the indications for surgical treatment of bone metastases in NSCLC. In this study, we examined the clinical benefit of surgical treatments, including laminectomy and posterolateral fusion, in patients with vertebral metastases from NSCLC, and evaluated the discrepancy between clinical outcome and prognosis as predicted by the Tokuhashi and Tomita scores prior to surgery.

\section{Patients and methods}

Patients. The clinical course of patients who received surgical treatment for neurological paralysis resulting from vertebral metastasis of advanced NSCLC at the Shiga University of Medical Science Hospital (Otsu, Japan) between April, 2007 and March, 2012 was retrospectively analyzed. All the patients provided written informed consent prior to surgery, and underwent laminectomy in combination with posterior spinal fixation surgery. After the surgical treatment, systemic anticancer therapy for bone metastasis, consisting of cytotoxic chemotherapeutic agents or molecular-targeted drugs and radiotherapy, was considered in patients whose PS and ADL improved.

Tokuhashi and Tomita scores. To predict the prognosis of patients with bone metastases, the Tokuhashi and Tomita scores were calculated preoperatively. The Tokuhashi scores range between 0 and 15 and are based on six criteria: PS (0: PS 3-4; 1: PS 2; and 2: PS 0-1); number of extraspinal bone metastases $(0: n \geq 3 ; 1: n=1-2$; and $2: n=0)$; number of spinal bone metastases $(0: n \geq 3 ; 1: n=2$; and $2: n=1)$; primary organ of cancer ( 0 : lung cancer); metastases in major internal organs $(0$ : unresectable; 1 : resectable; and 2: no metastases); and status of paralysis (0: Frankel A-B; 1: Frankel C-D; and 2: Frankel E). The prognosis of the patients is estimated as follows: 0-8, $\leq 6$ months; $9-11, \geq 6$ months; and 12-15, $\geq 12$ months (9). The Tomita score ranges from 2 to 10 and includes three criteria: Status of bone metastases (1: solitary or isolated; 2 : multiple); metastases in visceral organs (2: treatable; 4 : untreatable); and primary organ of cancer (4: lung cancer). The surgical strategy
Table I. Patient characteristics $(n=4)$.

\begin{tabular}{lc}
\hline Characteristics & $\mathrm{N}(\%)$ \\
\hline Median age, years (range) & $56.5(4167)$ \\
Gender & $3(75.0)$ \\
Male & $1(25.0)$ \\
Female & \\
Histology & $4(100.0)$ \\
Adenocarcinoma & \\
EGFR status & $2(50.0)$ \\
Mutated & $2(50.0)$ \\
Wild-type & \\
Symptoms associated with SREs & $3(75.0)$ \\
Pain & $4(100.0)$ \\
Paralysis & $4(100.0)$ \\
Pathological fractures & \\
\hline
\end{tabular}

EGFR, epidermal growth factor receptor gene; SREs, skeletal related events.

for the patients is recommended as follows: Score 2-3, wide or marginal excision; 4-5, marginal or intralesional excision; 6-7, palliative surgery; and 8-10, supportive care (10).

Statistical analysis. The clinical courses of the patients were obtained from their clinical records, and the median paralysisfree survival and overall survival (OS) after the fixation surgery were calculated according to the Kaplan-Meier method using SPSS 22.0 software (IBM, Armonk, NY, USA).

\section{Results}

Patient characteristics. Four patients with SREs attributable to advanced NSCLC were enrolled in this study (Table I). The patients included 3 men and 1 woman, with a median age of 56.5 years (range, 41-67 years). The histological type of NSCLC was adenocarcinoma in all patients, and 2 patients were found to have pulmonary adenocarcinoma with epidermal growth factor receptor gene (EGFR) mutation. The median time from the initial diagnosis of NSCLC to the onset of SREs was 1.5 months (range, 0-38 months). Of the symptoms commonly associated with SREs, pain, paralysis and pathological fractures were observed in 3,4 and 4 patients, respectively, whereas hypercalcemia was not observed in any of the patients. By the onset of SREs, 3 patients had been administered zoledronic acid. The median time from the onset of SREs to fixation surgery was 22.5 days (range, 10-25 days). The status of paralysis prior to fixation surgery was considered to be Frankel grade $\mathrm{C}$ for all patients, whereas the Eastern Cooperative Oncology Group PS was 2 in all patients.

Tokuhashi and Tomita scores prior to surgical fixation. The Tokuhashi score prior to surgical fixation was calculated to be 7 for 1 patient, 8 for 1 patient and 9 for 2 patients, with a median preoperative score of 8.3 . The data suggested a survival prognosis of $<6$ months in 2 patients and $<12$ months 
Table II.Clinical outcomes.

\begin{tabular}{|c|c|c|c|c|}
\hline Variables & Case 1 & Case 2 & Case 3 & Case 4 \\
\hline Regions of vertebral metastases & C5-Th2 & Th4-10 & Th4-10 & C5-Th6 \\
\hline Preoperative Tokuhashi score & 9 & 8 & 7 & 9 \\
\hline Preoperative Tomita score & 8 & 6 & 8 & 6 \\
\hline Frankel grade (before $\rightarrow$ after surgery) & $\mathrm{C} \rightarrow \mathrm{D}$ & $\mathrm{C} \rightarrow \mathrm{D}$ & $\mathrm{C} \rightarrow \mathrm{D}$ & $\mathrm{C} \rightarrow \mathrm{E}$ \\
\hline ECOG PS (before $\rightarrow$ after surgery) & $2 \rightarrow 1$ & $2 \rightarrow 1$ & $2 \rightarrow 1$ & $2 \rightarrow 1$ \\
\hline Postoperative therapy & $\begin{array}{c}\text { Chemotherapy } \\
\text { EGFR-TKI } \\
\text { Radiation }\end{array}$ & $\begin{array}{l}\text { Chemotherapy } \\
\text { EGFR-TKI } \\
\text { Bisphosphonate }\end{array}$ & $\begin{array}{l}\text { Chemotherapy } \\
\text { Radiation } \\
\text { Bisphosphonate }\end{array}$ & $\begin{array}{c}\text { Chemotherapy } \\
\text { EGFR-TKI } \\
\text { Bisphosphonate }\end{array}$ \\
\hline $\begin{array}{l}\text { Predicted prognosis by } \\
\text { Tokuhashi score (months) }\end{array}$ & $<12$ & $<6$ & $<6$ & $<12$ \\
\hline $\begin{array}{l}\text { Postoperative paralysis-free } \\
\text { period (months) }\end{array}$ & 17 & 43 & 40 & 42 \\
\hline Postoperative survival time (months) & 22 & 44 & 40 & 43 \\
\hline
\end{tabular}

PS, performance status; EGFR-TKI, epidermal growth factor receptor-tyrosine kinase inhibitor; OS, overall survival; ECOG PS, Eastern Cooperative Oncology Group performance status.

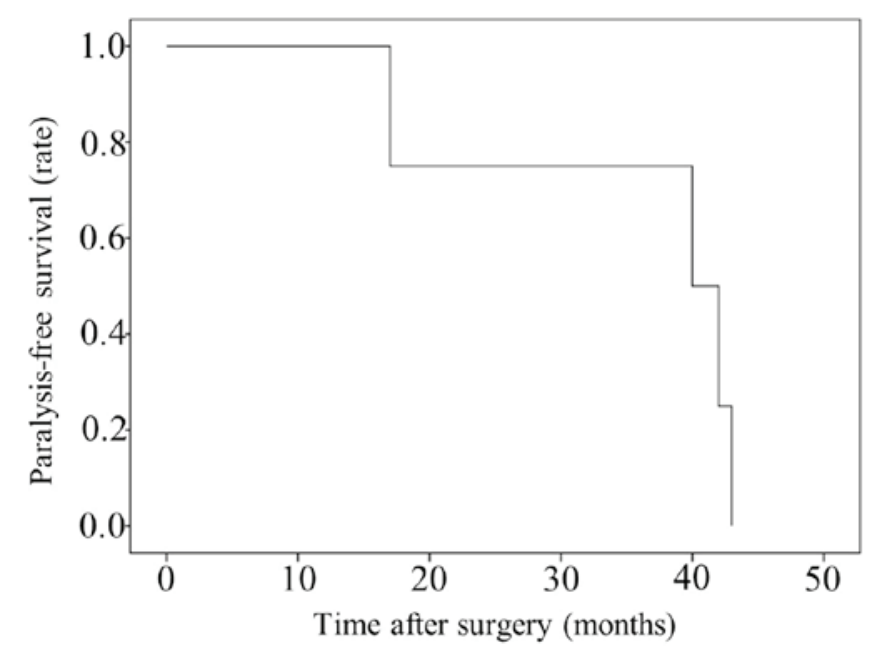

Figure 1. Paralysis-free survival rate following posterior fixation surgery in patients with vertebral metastasis from non-small-cell lung cancer.

in the other 2 patients. As regards the Tomita score prior to surgical fixation, 2 patients were calculated to have 6 points, indicating that palliative surgery was recommended; the remaining 2 patients were calculated to have 8 points, which was an indication for supportive care but not palliative surgery.

Clinical outcome of surgical fixation. All 4 patients underwent laminectomy in combination with posterior spinal fixation surgery, and experienced no complications associated with the operation. At a median time of 15 days after fixation surgery, the Frankel grade was D for 3 patients and E for 1 patient, demonstrating that the paralysis was improved by the surgery. Furthermore, the Eastern Cooperative Oncology Group PS of all patients was 1, demonstrating that the patients' ADL had also improved postoperatively. After the surgery, all the patients received several anticancer therapies; systemic

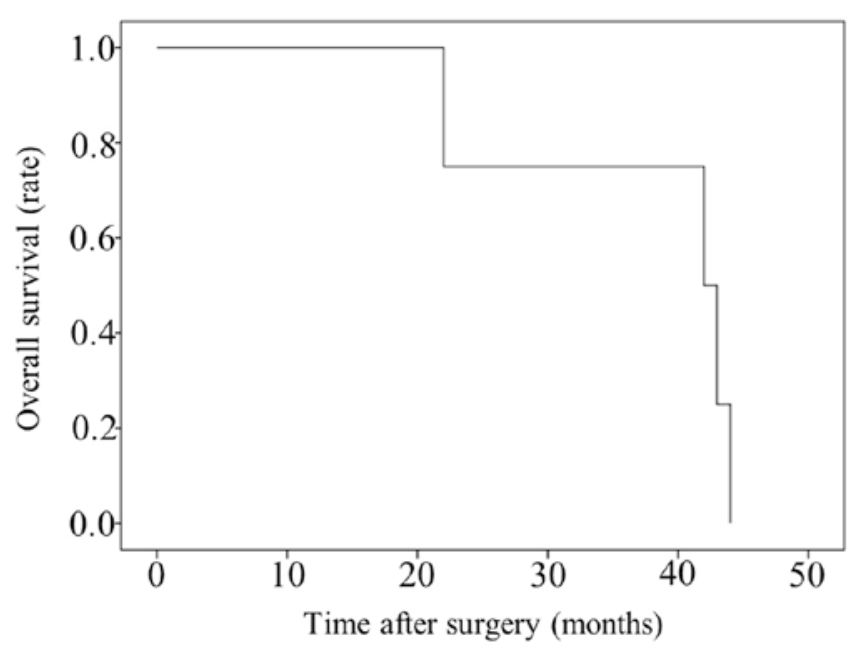

Figure 2. Overall survival rate following posterior fixation surgery in patients with vertebral metastasis from non-small-cell lung cancer.

chemotherapy was initiated in all 4 patients, EGFR-tyrosine kinase inhibitor (TKI) treatment in 4, and radiation therapy in 2 patients. The median paralysis-free time following fixation surgery was 41.0 months (range, 17-43 months; Fig. 1), and the median OS postoperatively was 42.5 months (range, 22-44 months; Fig. 2, Table II).

\section{Discussion}

In the present study, the clinical benefit of surgical treatment in patients with vertebral metastases arising from advanced NSCLC was reported. At the onset of SREs, these patients were predicted to have a poor prognosis as estimated according to the Tokuhashi score. However, surgical fixation improved the patients' ADL and enabled them to receive anticancer therapy, contributing to prolonged survival. 
It has been reported that bone metastasis develops in $30-60 \%$ of patients with advanced lung cancer. The most frequent site of bone metastasis are the vertebrae (12), comprising $>50 \%$ of all bone metastases from lung cancer (13). Bone metastasis may cause SREs such as intolerable pain, pathological fractures and paralysis, leading to deterioration of the patients' ADL, PS and QoL. Patients with SREs appear to miss the opportunity to receive active anticancer therapy due to their SRE-related deteriorating status. Thus, surgical intervention for SREs may improve the patients' condition and, subsequently, their ADL and QoL.

In this study, the clinical benefit of surgical treatments, including laminectomy and posterolateral fusion, was evaluated in patients with vertebral metastases from advanced NSCLC. The median OS of our patients following fixation surgery was 42.5 months. Taking into consideration the previously reported OS of 13.4 months for patients with bone metastases from lung cancer (14), our patients achieved a significantly prolonged survival. The surgical treatment for SREs contributed to the improvement of their PS and enabled them to receive subsequent anticancer (but not palliative) therapy, such as molecular-targeted therapy (EGFR-TKIs) and cytotoxic chemotherapy. It has been reported that EGFR-TKIs may prolong the OS of patients with EGFR-mutated NSCLC to 21.6-30.9 months (15-17). Surgical treatment of vertebral metastases from lung cancer provides the patient with the further opportunity to receive active moleculartargeted therapy or chemotherapy, thus contributing to the prolongation of OS.

To aid the decision-making process for the surgical treatment of bone metastases, the Tokuhashi score has been used to predict the prognosis of cancer patients (9). In this report, the suitability of this scoring system was evaluated in cases with advanced NSCLC with vertebral metastases. According to the Tokuhashi score, the survival prognosis of patients in the study was predicted to be $<6$ months for 2 patients and 6-12 months for the other 2 patients; however, their OS was in fact significantly longer compared with that predicted by the Tokuhashi score prior to surgery.

The reason why the predicted prognosis was poorer compared with the actual OS is associated with a specific criterion of the Tokuhashi score. The primary site of cancer, i.e., aggressiveness of cancer growth, is reflected in the score. Given that lung cancer is particularly aggressive, a lung cancer patient is given a low Tokuhashi score. Thus, based on this score the predicted survival of patients with NSCLC is estimated to be up to 12 months, which is consistent with the data of patients with bone metastases from lung cancer, reporting an OS of $\leq 6$ months $(12,18)$. However, the OS was recently reported to be prolonged up to 13.4 months (14) by the administration of EGFR-TKIs and newly developed chemotherapies.

In this context, the Tokuhashi score at present is likely unsuitable for the prediction of prognosis in patients with lung cancer and requires a revision. Hessler et al compared the predicted and achieved OS in 76 patients with vertebral metastases from lung cancer, reporting that they were similar in 51 of the 76 cases (67.1\%) and unequal in 25 of the 76 cases $(32.9 \%)$; they concluded that the Tokuhashi score was a suboptimal tool for the prediction of prognosis in patients with bone metastases from lung cancer (19). Given that their data were collected between 1999 and 2004, it is highly likely the patients in their study were not treated with EGFR-TKIs. We consider that a specific prognostic score for evaluating the prognosis of patients with bone metastases from NSCLC should be developed, including the criteria of genetic and histological types of lung cancer.

The Tomita score is also a decision-making tool with regard to surgical treatment for bone metastasis (10) when selecting a suitable treatment strategy. We evaluated whether the Tomita score would be applicable in cases of lung cancer with vertebral metastases. According to this scoring system, 2 of the 4 patients in the study were recommended to receive supportive care, but not palliative surgery. In fact, all our patients received palliative surgery, achieving a median OS after surgical fixation of 42.5 months. Both the Tomita and Tokuhashi scores are influenced by the primary organ of cancer. Therefore, the Tomita score generally recommends that patients with NSCLC who have metastases in the bone as well as other organs should receive supportive care. However, if the clinical condition of the patients is improved by palliative surgery, they are given the opportunity to receive active anticancer therapy. Aoude et al reported that surgical treatment for vertebral metastases of lung cancer was decided upon when the Tomita score was 5 (20). However, in patients with lung adenocarcinoma with EGFR mutation, the prognosis may improve by administration of EGFR-TKIs.

In conclusion, surgical treatment, including fixation surgery, may be beneficial for a proportion of patients with neurological paralysis caused by vertebral metastasis of advanced NSCLC. A specific score suggesting the appropriate treatment for patients with bone metastases from advanced NSCLC should be developed, incorporating criteria in relation to the genetic and histological types of lung cancer.

\section{References}

1. Stewart BW and Wild CP: World Cancer Report. IARC, Lyon, 2014.

2. Silvestri GA, Tanoue LT, Margolis ML, Barker J and Detterbeck F; American College of Chest Physicians: The noninvasive staging of non-small cell lung cancer: The guidelines. Chest 123 (Suppl): 147S-156S, 2003.

3. Peters S, Weder W, Dafni U, Kerr KM, Bubendorf L, Meldgaard P, O'Byrne KJ, Wrona A, Vansteenkiste J, Felip E, et al; ETOP Lungscape Investigators: Lungscape: Resected non-small-cell lung cancer outcome by clinical and pathological parameters. J Thorac Oncol 9: 1675-1684, 2014.

4. Wong DA, Fornasier VL and MacNab I: Spinal metastases: The obvious, the occult, and the impostors. Spine 15: 1-4, 1990.

5. Weigel B, Maghsudi M, Neumann C, Kretschmer R, Müller FJ and Nerlich M: Surgical management of symptomatic spinal metastases. Postoperative outcome and quality of life. Spine 24: 2240-2246, 1999.

6. Schaberg J and Gainor BJ: A profile of metastatic carcinoma of the spine. Spine 10: 19-20, 1985.

7. Henry DH, Costa L, Goldwasser F, Hirsh V, Hungria V, Prausova J, Scagliotti GV, Sleeboom H, Spencer A, Vadhan-Raj S, et al: Randomized, double-blind study of denosumab versus zoledronic acid in the treatment of bone metastases in patients with advanced cancer (excluding breast and prostate cancer) or multiple myeloma. J Clin Oncol 29: 1125-1132, 2011.

8. Walker MP, Yaszemski MJ, Kim CW, Talac R and Currier BL: Metastatic disease of the spine: Evaluation and treatment. Clin Orthop Relat Res 415 (Suppl): S165-S175, 2003.

9. Tokuhashi Y, Matsuzaki H, Toriyama S, Kawano H and Ohsaka S: Scoring system for the preoperative evaluation of metastatic spine tumor prognosis. Spine 15: 1110-1113, 1990.

10. Tomita K, Kawahara N, Kobayashi T, Yoshida A, Murakami H and Akamaru T: Surgical strategy for spinal metastases. Spine 26: 298-306, 2001. 
11. Frankel HL, Hancock DO, Hyslop G, Melzak J, Michaelis LS Ungar GH, Vernon JD and Walsh JJ: The value of postural reduction in the initial management of closed injuries of the spine with paraplegia and tetraplegia. I. Paraplegia 7: 179-192, 1969.

12. Coleman RE: Clinical features of metastatic bone disease and risk of skeletal morbidity. Clin Cancer Res 12: 6243s-6249s, 2006.

13. Tsuya A, Kurata T, Tamura K and Fukuoka M: Skeletal metastases in non-small cell lung cancer: A retrospective study. Lung Cancer 57: 229-232, 2007.

14. Deberne M, Ropert S, Billemont B, Daniel C, Chapron J and Goldwasser F: Inaugural bone metastases in non-small cell lung cancer: A specific prognostic entity? BMC Cancer 14: 416, 2014

15. Mok TS, Wu YL, Thongprasert S, Yang CH, Chu DT, Saijo N, Sunpaweravong P, Han B, Margono B, Ichinose Y, et al: Gefitinib or carboplatin-paclitaxel in pulmonary adenocarcinoma. N Engl J Med 361: 947-957, 2009.

16. Mitsudomi T, Morita S, Yatabe Y, Negoro S, Okamoto I, Tsurutani J, Seto T, Satouchi M, Tada H, Hirashima T, et al; West Japan Oncology Group: Gefitinib versus cisplatin plus docetaxel in patients with non-small-cell lung cancer harbouring mutations of the epidermal growth factor receptor (WJTOG3405): An open label, randomised phase 3 trial. Lancet Oncol 11: 121-128, 2010.
17. Maemondo M, Inoue A, Kobayashi K, Sugawara S, Oizumi S, Isobe $\mathrm{H}$, Gemma A, Harada M, Yoshizawa H, Kinoshita I, et al; North-East Japan Study Group: Gefitinib or chemotherapy for non-small-cell lung cancer with mutated EGFR. N Engl J Med 362: 2380-2388, 2010

18. Decroisette C, Monnet I, Berard H, Quere G, Le Caer H, Bota S, Audigier-Valette C, Geriniere L, Vernejoux JM and Chouaid C; Groupe Français de Pneumo-Cancérologie 0601 Team: Epidemiology and treatment costs of bone metastases from lung cancer: A French prospective, observational, multicenter study (GFPC 0601). J Thorac Oncol 6: 576-582, 2011.

19. Hessler C, Vettorazzi E, Madert J, Bokemeyer C and Panse J: Actual and predicted survival time of patients with spinal metastases of lung cancer: Evaluation of the robustness of the Tokuhashi score. Spine 36: 983-989, 2011.

20. Aoude AA and Amiot LP: Surgical treatment of a patient with lung cancer metastasized to the spine with EGFR mutation: A case report. Int J Surg Case Rep 3: 510-512, 2012. 